\title{
Lecture des espaces en mouvement : géocritique et cartographie
}

\section{Bertrand Westphal}

\section{(2) OpenEdition}

\section{Journals}

Édition électronique

URL : http://journals.openedition.org/edl/478

DOI : $10.4000 /$ edl. 478

ISSN : 2296-5084

Éditeur

Université de Lausanne

\section{Édition imprimée}

Date de publication : 15 mai 2013

Pagination : 17-32

ISBN : 978-2-940331-32-1

ISSN : 0014-2026

\section{Référence électronique}

Bertrand Westphal, «Lecture des espaces en mouvement : géocritique et cartographie », Études de lettres [En ligne], 1-2 | 2013, mis en ligne le 15 mai 2016, consulté le 19 décembre 2020. URL : http:// journals.openedition.org/edl/478; DOI : https://doi.org/10.4000/edl.478 


\section{LECTURE DES ESPACES EN MOUVEMENT: GÉOCRITIQUE ET CARTOGRAPHIE}

Quelles qu'en soient les modalités, la carte géographique est un objet de constante fascination pour les artistes. Pour s'en convaincre, on partira de quatre exemples: les tapisseries murales à sujet cartographique qui ornent le mur de plusieurs intérieurs bourgeois peints par Vermeer; les spéculations cartographiques que Christopher Marlowe prête au conquérant Tamerlan; les propos hautement politiques que des géographes militaires britanniques imaginés par le dramaturge Brian Friel échangent avec les habitants d'un petit village irlandais dont les toponymes gaéliques sont destinés à être anglicisés; et, pour revenir au présent, les œuvres à mi-chemin entre planches anatomiques et planches d'atlas conçues par Kathy Prendergast, artiste irlandaise. A partir de ces différentes illustrations (au sens fort), on se livrera à une réflexion sur les liens possibles entre cartes, textes et images, dans une dimension géocritique intégrant des excursus postcoloniaux et gender.

Depuis longtemps, la carte occupe le terrain et couvre les recoins les plus énigmatiques du globe, comme un reporter couvre les grands événements du monde. Elle vaut qu'on en parle. Concentrons-nous pour commencer sur quelques exemples et déplaçons-nous, car parler d'espace suppose que l'on franchisse les limites de la pure formalisation théorique. Et, avec un peu d'imagination, voyageons aussi dans le temps.

La première des quatre étapes de ce périple nous mènera à Delft, entre 1655 et 1675, où nous rencontrerons peut-être le grand Jan Vermeer - "peut-être", disais-je car l'homme préférait l'intimité des maisons à pignon plutôt que la fréquentation des rues ou les promenades au grand air dans les champs où commençaient à fleurir les tulipes importées de Turquie. Vermeer avait beau affectionner les pièces cossues des maisons de sa ville natale, il vouait un véritable culte aux cartes - aux cartes 
géographiques, symboles de l'ouverture sur le lointain. Vermeer et les cartes, donc... A quels tableaux penser? Pourquoi pas à L'Astronome ou au Géographe, l'un incliné sur un globe, l'autre penché sur un atlas et tel rouleau de cartes dépliées. Mais ce serait presque trop facile, joué d'avance, scontato, comme disent les Italiens. Prenons plutôt L'Officier et la jeune fille riant, La jeune Femme à l'aiguière, La femme en bleu lisant une lettre, La Femme au luth, L'Allégorie de la peinture. Tous ces chefsd'œuvre, et j'en omets plusieurs, présentent un point commun, a priori inattendu. Certes, tous dévoilent devant nos yeux le plan en coupe d'un intérieur bourgeois de la bonne ville de Delft illuminé par des rais de lumière que tamisent les vitres d'une fenêtre entrouverte (on la voit parfois sinon on la devine). C'est en quelque sorte la marque de fabrique du grand Vermeer. Mais ce qui nous intéressera ici au premier chef, c'est que tous exposent à l'arrière-plan une mappemonde suspendue au mur en guise de tapisserie. On ajouterait volontiers que si l'astronome et le géographe des tableaux sont des hommes, les figures qui jouxtent les différentes tapisseries cartographiques sont invariablement des femmes ${ }^{1}$. Sans être historien de l'art et expert de l'œuvre de Vermeer, je sais pour l'avoir lu - mais je crois même que je l'aurais deviné - que la plupart de ces compositions tendent à établir un contraste saisissant entre d'une part le sentiment de confort qu'inspire l'intérieur douillet situé à l'abri des digues et d'autre part le rêve de lointain ou pour le moins le désir d'ailleurs qu'exprime le regard songeur que la jeune femme lance en direction de la fenêtre, quelques secondes peut-être - allez savoir! - après avoir contemplé la mappemonde accrochée à la cloison. Rien n’empêche de penser que Vermeer fût le premier à coupler la carte et le rêve, car il

I. L'œuvre de Vermeer ne constitue de loin pas une exception. Dans plusieurs essais, dont Atlas of Emotion de G. Bruno et Mapping Discord de J. N. Peters, sont mentionnés des tableaux où des figures féminines sont associées à des cartes ou à des globes. Deux exemples seulement, tirés du beau livre de G. Bruno, La lezione di geografia (1752) de Pietro Longhi qui met en scène une élégante jeune femme commentant une carte en présence de deux messieurs et de servantes apportant le café (aussi exotique que certaines régions esquissée sur les cartes) et le frontispice de l'Atlas (1792) de Jan Barend Elwe qui représente des femmes dessinant le globe sous l'œil attentif d'Atlas. On n'oubliera pas que Vermeer ne fut pas le seul à suspendre des cartes dans ses toiles, en Hollande. Entre la fin des années 1660 et le début des années 1680, Jacob Ochtervelt, peintre originaire de Rotterdam, a réalisé plusieurs tableaux associant de jeunes femmes à des clavecins et des cartes tapissées suspendues au-dessus de l'instrument (voir à ce sujet S. Alpers, "The Mapping Impulse in Dutch Art»). 
faut bien reconnaître qu'avant lui, et après lui aussi bien, celle-là a surtout reflété ou suscité l'avidité et l'acharnement des hommes.

C'est justement une leçon sur l'avidité qui va motiver notre deuxième étape, qui intervient plus tôt dans l'Histoire et plus loin de l'Europe, sous une tente à Otrar, dans le Kazakhstan actuel, en 1405. Tamerlan, qui s'apprêtait à attaquer la Chine des Ming, a contracté la peste quelques semaines auparavant. Il agonise quelque part dans la steppe alors qu'il s'est emparé d'une bonne portion du monde de son époque. D’une portion, d'une large portion, mais pas de l'ensemble. Il lui manquait en effet l'un ou l'autre territoire pour remporter la partie de Risk qu'il avait engagée. Pas le Kamtchatka, péninsule de l'Extrême-Orient russe que le célèbre jeu de société, si violent dans son dépouillement, a popularisé. Mais d'autres territoires qu'évoquera Christopher Marlowe dans la pièce qu'il consacra en 1587 au chef de guerre mongol. Sur son lit de mort, Tamerlan s'enquiert d'une carte. On la déroule; il entreprend de dresser l'inventaire des conquêtes qui l'ont conduit de Samarcande jusqu'à Zanzibar et jusqu'en Perse. Il pointe son doigt sur des aires vierges et s'écrie: "Je meurs, rien de cela n'est conquis!" ${ }^{2}$. Son dépit est aussi grand que le fut naguère son appétit de conquête. J'ignore si Tamerlan recourait vraiment à des cartes. Sans doute que si, même si la cartographie, en ces temps-là, n'était pas l'affaire des Mongols et des Chinois. Les cartes que Marlowe confie à son héros, si elles inspiraient bien un désir d'ailleurs, matérialisent avant tout une appropriation en cours, une cible géographique. Frustré davantage par la tâche laissée en suspens que par l'imminence de sa mort, Tamerlan se tourne vers ses enfants: "Mes fils, voyez ces étendues de terres / A mi-chemin du Cancer jusque vers l'ouest» ${ }^{3}$. Implicitement, il leur indique qu'il leur appartiendra de s'emparer des derniers territoires manquant au grand empire. Ils échoueront.

La troisième étape est plus proche de nous à la fois dans le temps et dans l'espace. Elle nous vaut une halte à Baile Beag ou Ballybeg, dans le comté de Donegal, en Irlande, en août 1833. Parler de ce village dans le cadre d'une étude sur la cartographie revêt une dimension paradoxale, car Baile Beag ou Ballybeg ne figure sur aucune carte. Il s'agit d'un nom générique qui, dans la verte Erin, signifie "petite bourgade».

2. Ch. Marlowe, Tamerlan (2, V, 3, 159), p. 243. En anglais, la formule est plus percutante: "And shall I die, and this unconquered?"

3. Ch. Marlowe, Tamerlan (2, V, 3, 146-147), p. 243. 
Baile Beag/Ballybeg est le lieu fictionnel qui sert de décor habituel, de set comme on dirait à Hollywood, à l'un des plus grands dramaturges contemporains: l'Irlandais Brian Friel. En août 1833, quelques élèves sont réunis autour de Hugh O'Donnell, maître de latin et de grec, qui aime lever le coude plus que de raison. Ses deux fils, Manus et Owen, se trouvent également dans l'assistance. Owen est revenu de Dublin en compagnie de deux officiers anglais, le capitaine Lancey, cartographe de son état, et le lieutenant Yolland, expert en toponymie. Leur mission consiste à angliciser les noms gaéliques, à faire en sorte que Baile Beag devienne un Ballybeg crédible et conforme aux usages de la Couronne. Owen, qui se fait appeler Roland, charité bien ordonnée commençant par soi-même, leur servira d'intermédiaire, de truchement linguistique car les uns et les autres ne se comprennent pas: c'est au point que lorsque Hugh entreprend de parler latin aux militaires ceux-ci croient entendre du gaélique. Mais les malentendus linguistiques ne sont rien comparés aux difficultés diplomatiques, car on imagine aisément que l'application des ordres vaudra quelque contrariété aux zélés militaires, surtout lorsqu'ils expliquent à leurs interlocuteurs que les relevés serviront à lever des taxes. Voilà en quelques mots le cadre général de Translations, une pièce de 1981 qui, à ma connaissance, n’a pas été traduite en français, malgré un titre propice. Cahin-caha, on effectue les modifications toponymiques, que l'on mime les consonances gaéliques en anglais ou que l'on traduise les étymons (ce qui ne vas pas toujours de soi, car il arrive que le sens du toponyme se soit égaré en cours de route, si je puis dire). Le lieutenant Yolland manifeste parfois de la curiosité à l'égard d'une culture qu'il découvre, mais il finit par disparaître, ce qui attire sur les villageois de sévères menaces de représailles de la part du capitaine Lancey. Lorsque celui-ci mentionne en anglais les emplacements des maisons qui seront rasées si on ne délivre pas son subalterne, Owen/ Roland traduit au fur et à mesure en gaélique. La boucle est ainsi bouclée. Owen, qui cesse de se faire appeler Roland, a compris que l'avertissement de son frère Manus, à la fin du premier acte était fondé: «It's a bloody military operation, Owen!» ${ }^{4}$. Plusieurs habitants de Baile Beag avaient fait preuve à l'égard du colonisateur anglais de la même candeur que les Tainos, premiers peuples des Caraïbes, à l'égard de Colomb et de ses successeurs hidalgos. Comme eux, ils ont déchanté.

4. B. Friel, Translations, p. 408. 
Il eût été dommage de ne pas réserver une quatrième et dernière étape aux Etats-Unis. Au demeurant, elle aurait pu être imprégnée d'Irish Spirit. J'aime en effet à citer l'œuvre de Kathy Prendergast, une artiste irlandaise, qui allie comme Vermeer, mais selon des modalités fort différentes, cartographie et peinture. Kathy Prendergast a reporté sur l'Amérique du Nord le processus de déperdition que Brian Friel a décrit en Irlande. Pour ce faire, elle a peint une toile qui s'intitule Lost (1999). Elle représente une carte des Etats-Unis et du Canada qui ne reproduit qu'une seule série de toponymes: ceux qui comportent l'épithète Lost. Seul ce qui est perdu reste visible. A vrai dire, j'ai longtemps cru que ces noms renvoyaient à la géographie objective, mais, vérification faite, il n'en est que trois aux Etats-Unis: Lost Creek et ses 467 âmes en Virginie occidentale, Lost Hills et ses 1938 habitants en Californie et Lost Nation et ses 497 résidents dans l'Iowa, contre aucun Lost canadien. C'est qu'on n'aime guère revenir sur ce que l'on a égaré ou, plus exactement, sur ce que l'on a fait perdre aux autres - ce qui, traduit en clair, revient à dire: ce dont on les a dépouillés.

Restons encore un instant aux Etats-Unis. Chez un autre créateur contemporain, Michael Chabon, il n'est pas question d'une perte mais au contraire d'un remplissage indu. Chabon, qui est l'un des principaux écrivains américains de la jeune génération, est né à Washington en 1963. Comme ses parents divorcèrent alors qu'il était enfant, il passa une partie de son adolescence à Pittsburgh, chez son père, et l'autre à Columbia chez sa mère. Ce Columbia-là n'a rien à voir avec l'allégorie bien connue et à peine plus avec l'université new-yorkaise: il s'agit d'une ville nouvelle fondée dans les années soixante au Maryland. Chabon en parle dans le récit qui donne son titre au recueil Maps and Legends, publié en 2009, et confie son adresse d'alors à ses lecteurs: 5179 Eliots Oak Road, dans le Neighborhood de Longfellow situé dans le Village de Harper's Choice. Harper's Choice doit son nom à un agent immobilier dont Harper était le prénom; c'est peut-être le signe que l'imagination de l'agent chargé de la toponymie des lieux avait fini par tarir; c'est en tout cas l'hypothèse de Chabon. Pour leur part, les noms de la rue et du quartier sont plus littéraires. Si l'on connaît Longfellow, on ne saura pas nécessairement que l'un de ses poèmes est intitulé Eliots Oak. Toutes les rues du quartier portent le nom de titres tirés de l'œuvre du poète de Portland, dans le Maine, des titres peut-être plus familiers aux Américains qu'à nous. De fait, le plan d'occupation des sols avait prévu que Columbia serait divisé 
en Villages, eux-mêmes subdivisés en Neighborhoods. Et Chabon de commenter:

Ces Villages avaient tous été conçus et nommés; ils étaient présents sur la carte et délimités [...]. Mais, sur cette carte, il y avait de vastes secteurs qui, à l'exception du nom du Village, étaient complètement vides et conjecturaux, inexistants, de fait 5 .

La carte pointait donc des lieux virtuels. Ce qui inspire à Chabon un commentaire supplémentaire, digne d'un futur écrivain: "A mes yeux, il s'agissait d'une puissante démonstration du pouvoir incantatoire des noms et de la nomination. " ${ }^{6}$ Là encore, je suis allé vérifier sur une carte actuelle quel sort a été réservé à Columbia et à ses rues fantômes. Que l'on se rassure, tout ce qui a été envisagé sur le papier a fini par s'accomplir. La ville compte aujourd'hui près de cent mille habitants.

La fin de ce voyage en quatre étapes à travers le pays des cartes intervient ici. Comme tout voyage, il s'accompagne de quelques leçons assez instructives. Que constatons-nous en effet? Que la cartographie fait bon ménage avec la littérature, dont elle constitue une métaphore privilégiée. Les quelques exemples égrenés ci-dessus ne sont pas isolés. Du Voyage de Gulliver à Absalom, Absalom!, de L'Ile au trésor au Seigneur des Anneaux, les cartes ne cessent de se déployer au détour des pages. Et encore, pour une raison qui m'échappe, ai-je privilégié ici la littérature anglophone. Mais est-ce vraiment dû au hasard? Si j'en crois Gilles Deleuze, qui dans ses dialogues avec Claire Parnet a prêché «la supériorité de la littérature anglaise-américaine» (diantre!), tout du moins en matière de spatialité, ce réflexe n'est pas nécessairement fortuit:

La littérature américaine opère d'après des lignes géographiques: la fuite vers l'ouest, la découverte que le véritable Est est à l'Ouest, le sens des frontières comme quelque chose à franchir, à repousser, à dépasser. Le devenir est géographique. On n'a pas d'équivalent en France. Les

5. M. Chabon, Maps and Legends, p. 18: "These Villages had all been laid out and named, and were present on and defined by the map [...]. But there were large areas of the map that, apart from the Village name, were entirely empty, conjectural non-existent, in fact."

6. M. Chabon, Maps and Legends, p. 19: «It was a powerful demonstration to me of the incantatory power of names and naming.» 
Français sont trop humains, trop historiques, trop soucieux d'avenir et de passé. Ils passent leur temps à faire le point ${ }^{7}$.

Certes, cette assertion - comme tout ce qui tend à comparer des cultures, à quérir leur essence - relève de l'inépuisable catégorie des jugements à l'emporte-pièce et on pourrait soupçonner Deleuze d'avoir regardé trop de westerns à la télévision, mais elle n'est pas totalement dépourvue de fondement. La traversée des grands Etats de l'Ouest ou du Sud-Ouest américain arrachent une adhésion au pur espace que peu d'endroits d'Europe, et de France en particulier, sont en mesure de conforter. Notre horizon est sans cesse troublé, au sens premier du terme, autant qu'au sens figuré: le regard éprouve les pires difficultés à balayer un espace vierge d'obstacles.

Mais revenons-en aux cartes. Si l'on s'astreint à un surcroît de discipline, on parviendra à un point crucial: celui de l'interdisciplinarité. Cartes et livres font bon ménage, disais-je. Mais ce lien débouche sur des couplages multiples. Par le biais des cartes, textes et toiles communiquent (Marlowe et Vermeer, Friel et Prendergast), comme si à chaque fois que des questions de représentation spatiale se posaient, les divers supports artistiques/fictionnels (littérature et peinture, mais aussi cinéma, photographie, etc.) pouvaient être convoqués de concert, presque indifféremment. Et ce débordement générique, catégorique, disciplinaire ne se borne pas aux arts. La géographie et son discours censé parler de la réalité objective se manifeste aussi, de même que l'urbanisme - ce qui n’a pas échappé à Michael Chabon, scrupuleux observateur du plan d'occupation des sols de Columbia. Parler des représentations spatiales, c'est soumettre le périmètre idéal de l'étude à une prolifération disciplinaire dont on ne maitrise jamais l'ensemble des implications. Les carrefours sont trop nombreux, les points d'inflexion multiples, virtuellement infinis: il convient de faire preuve d'humilité et de s'incliner devant l'indéfinissable... qui nous préserve de la saturation des perspectives. Voilà un moindre mal, car, en ce qu'elle résulte d'un effort inscrit dans la durée, dans la succession des strates d'analyses, l'herméneutique spatiale - comme toute herméneutique - se nourrit des lacunes de nos possibilités d'expression, de l'extrême relativité des résultats auxquels nous pouvons

7. G. Deleuze, C. Parnet, Dialogues, p. 48. 
prétendre dans l'instant. Toute thèse restera à jamais une hypothèse: bien présomptueux qui affirmera le contraire!

Autre indication qui découle de notre petit voyage de tout à l'heure: la carte mobilise des discours eux-mêmes très variés. Elle est avant tout l'expression d'une tentative de réduire le monde à une échelle compatible avec le point de vue humain, dont Pascal avait bien montré qu'il était relatif dans ses attendus (qu'est en effet la vérité des lignes de part et d'autre des Pyrénées?) et conventionnel (qu'est l'univers humain, sinon un milieu acceptable, tout au mieux honorable, entre le rien et le tout?). Pour les plus bravaches, l'échelle des cartes est l'instrument qui consent à l'homme de se portraiturer en dieu surplombant un univers dont il aurait la totale maîtrise. Un univers rond comme un globe ou plat comme une mappemonde. Hélas pour l'audacieux, il sera toujours objet d'un regard le surprenant en train de regarder la carte. Le géographe de Vermeer se sera-t-il cru divin, l'espace de quelques secondes ou de quelques minutes? Qu'importe, car une divinité supérieure (le peintre) a inclus la scène dans un théâtre plus vaste: celui de la toile. En proie à de semblables mises en abîme, les échappées de l'orgueil paraissent bien dérisoires. Tout ce qui semble grand l'est en proportion d'une échelle. Et fût-elle dénuée d'orgueil, cette posture resterait vaine, comme l'a compris Jorge Luis Borges qui tant de choses a comprises:

Nosotros (la indivisa divinidad que opera en nosotros) hemos soñado el mundo. Lo hemos sońado resistente, misterioso, visible, ubicuo en el espacio y firme en el tiempo; pero hemos consentido en su arquitectura tenues y eternos intersticios de sinrazón para saber que es falso ${ }^{8}$.

La carte et le globe sont remplis de ces interstices. Quant au géographe hollandais, sa fugitive impression aurait été exaltante. Sans doute lui aurait-il plu de se ménager, coûte que coûte, ce moment de surplomb intellectuel! Le Tamerlan de Marlowe devait avoir éprouvé un sentiment du même ordre, mais plus cru. Après tout, c'est à lui, comme à quelques autres, qu’a échu la responsabilité de modifier le dessin des cartes. Mais en vain, car Tamerlan agonise dans le regret, dans l'inassouvissement de sa faim inextinguible. La carte du monde est souvent la carte d'une hybris. On s'évertue un temps à remplir les blancs qui la parsèment (ces blancs n'étant du reste des blancs que dans la perspective du conquérant

8. J. L. Borges, Discusión, p. 171. 
en aucun cas les interstices qu'évoque Borges) ou alors on s'efforce de la ramener à la couleur que l'on aura choisie au détriment de toutes celles qui symbolisent la différence, une altérité. Ce temps sera plus ou moins long, plus ou moins bref, mais il sera toujours circonscrit. Au terme de l'intervalle, les pleins se vident à nouveau et les couleurs retrouvent leurs nuances. A la longue, la palette chromatique finira toujours par s'imposer dans son immense diversité. L' hybris ne mène à rien, à rien de durable. L'histoire des cartes, comme celle de la littérature et du genre tragique, en porte un témoignage inlassable.

Marlowe ne s'était pas montré si injuste à l'égard du conquérant. Tamerlan était incidemment une hypostase de sir Francis Drake, premier marin anglais qui effectua la circumnavigation des océans, quelques années à peine avant la première de la pièce. Il est même probable que Marlowe ait rendu justice à Tamerlan et qu'il ait contribué à en faire une figure littéraire défiant le passage des générations. En revanche, le dramaturge anglais a triché sur un point important en confiant la lecture d'une carte à son protagoniste. La cartographie était peu connue des Mongols, au tournant du XV siècle. Elle était occidentale, avant tout, à la fin du Moyen Age et davantage encore au cœur du baroque, que frôlait la scène élisabéthaine. On pourra certes se prévaloir d'une carte chinoise (en réalité, coréenne) de 1402, la carte Kangnido, qui précédait de cinquante-cinq ans la fameuse carte de Fra Mauro et qui, déjà, indiquait le pourtour de l'Afrique. On pourra aussi bien rappeler que les Chinois avaient tracé des cartes bien supérieures à celles des érudits grecs au $\mathrm{V}^{\mathrm{e}}$ siècle avant notre ère. On pourra même faire remarquer que les Polynésiens habitant l'archipel des îles Marshall disposaient de cartes maritimes impressionnantes depuis des époques reculées, les «stick charts", impressionnantes en raison des connaissances véhiculées, mais aussi, de leur forme: des bâtonnets de noix de coco assemblés en vue de tracer des itinéraires maritimes.

En tout état de cause, ces documents n'avaient rien de comparable avec les croquis européens du monde. C'est que pour l'Europe, la carte était le reflet iconographique d'une parcelle de monde promise à une appropriation prochaine. Elle préludait et accompagnait un envahissement, un débordement. Elle était le propre de l'Europe moderne, qui l'avait érigée en symbole - la modernité étant conçue, du début à sa fin, comme âge de la colonisation (Walter Mignolo). La carte était une imago mundi. En tant qu'image, son impact était considérable, or 
la tentation de cerner, voire d'orienter cet impact sur l'observateur était l'un des enjeux majeurs de l'époque. Le baroque a déterminé l'essor de l'image, de la colonisation des esprits et de la modernité qui encadre ce processus. Voici un peu plus de vingt ans, l'historien Serge Gruzinski a consacré un très bel essai à cette "guerre des images" (qui donne son titre à son livre). Comme souvent, en ce qui le concerne, son laboratoire est le Mexique colonial et baroque, qui a servi de théâtre à un combat acharné: celui que menèrent les Espagnols pour imposer leurs images saintes aux Aztèques. Voici ce qu'écrit Gruzinski à ce propos:

Si l'image oppose tant d'écueils, c'est qu'elle est la manifestation d'une structure qui la dépasse de toute part, l'expression d'un ordre visuel et plus encore d'un imaginaire dont l'assimilation consciente et inconsciente est synonyme d'occidentalisation [...]. Il ne s'agit pas seulement de la découverte d'un répertoire iconographique inédit mais de l'imposition de ce que l'Occident entend par personne, divin, nature, causalité, espace et histoire. En fait, sous les grilles stylistiques et perceptuelles opèrent d'autres grilles composant une armature conceptuelle et affective qui organise inconsciemment toutes les catégories de notre rapport au réel ${ }^{9}$.

En lisant Gruzinski, m'est revenue à l'esprit l'analyse sémiotique très caustique que Roland Barthes avait faite dans Mythologies, à la fin des années cinquante, de la photographie d'un tirailleur sénégalais saluant un drapeau tricolore (les trois couleurs de la France, pas encore celles du Sénégal). Ou de la bataille des images, des symboles, des signes, sur horizon de colonialisme triomphant chez Gruzinski, agonisant chez Barthes. Au moins autant que l'image pieuse d'une part et le cliché nationaliste de l'autre, la carte, la plupart des cartes, visent à orienter le spectacle d'un monde idéalement placé sous le projecteur occidental.

Il est exclu ici de développer plus avant cette réflexion qui ailleurs remplit des traités entiers. Je me contente donc d'en revenir une nouvelle fois aux quelques illustrations qui servent d'appoint à mon raisonnement. L'histoire de la lecture et de l'interprétation des cartes constitue à coup sûr l'un des paramètres de l'étude postcoloniale. Mais le postcolonial ne concerne pas seulement le récit du retour d'un territoire géographique

9. S. Gruzinski, La guerre des images de Christophe Colomb à Blade Runner (14922019), p. 131. 
à ses propriétaires légitimes. Des propriétaires légitimes spoliés de leur bien, il y en a quelques-uns. Ou mieux: quelques-unes! Comme je l'ai noté plus tôt, la carte, via la peinture, établit une connexion qui mérite d'être explorée avec le territoire féminin (Kathy Prendergast, notamment la série Body Map de 1983) ou l'espace du rêve féminin (Jan Vermeer). En l'occurrence, la carte porte le témoignage en creux d'un désir ou d'une revendication dont l'accomplissement est malaisé: vivre ailleurs, vivre son propre espace, vivre son propre corps. Le globe ou la mappemonde tendent à se transformer en marqueurs de restrictions paradoxales dont les figures féminines sont les objets. Soit le vaste monde pointé par le cartographe est là-bas, au-delà, hors de toute emprise matérielle et la femme est ici, engoncée dans un intérieur bourgeois, belle et innocente captive soustraite au regard extérieur (Vermeer devancerait alors Flaubert plaçant Emma Bovary derrière les carreaux de sa fenêtre); soit le vaste monde que pointe la carte, et qui a été conquis aux dépens d'autrui le plus souvent, est un monde masculin, dont la femme est absente - ou pire: dans lequel elle est présente sous forme d'oripeau, en tant qu'emblème d'une conquête assimilant la terre à une matrice et la prise de possession à une séduction dont l'issue fatale serait la reddition.

La carte associe l'image et le texte dans une perspective interdisciplinaire. Par ailleurs, la carte est la flatteuse expression d'une supériorité autoproclamée de l'homme face aux dieux, de l'homme face à la femme et de l'homme face aux autres hommes (et femmes) - une vaine et périlleuse posture dont maintes œuvres littéraires font le récit. Voilà qui a été évoqué. Il est une autre piste qui se dégage du corpus délibérément arbitraire que j’ai circonscrit à la faveur de cet article. La carte est un agencement de texte, ce que Friel et Chabon ont très bien perçu. La carte ne se contente pas de décrire l'espace planétaire sur le mode pseudo-objectif qui sous-tend et régit l'approche de nombreuses sciences humaines (humaines, oui... voire trop humaines); elle l'écrit - car la description qu'elle propose est un indéniable acte d'écriture ou de récriture et donc une entreprise fondatrice. L'Irlande existait avant la colonisation anglaise et les répressions qui l'ont rythmée. Ses toponymes étaient alors gaéliques. Lorsque, procédant à l'exécution des ordres, Lancey et Yolland nomment les lieux, ils les renomment et, par là même, dénomme ce qui préexistait. Tous les conquérants, tous les occupants se sont passionnément livrés à des tâches analogues avant eux. Que l'on songe aux Espagnols dans les Caraïbes et au Mexique, aux Français en 
Afrique, quelques siècles plus tard, mais aux Britanniques aussi bien, et aux Portugais avant tout le monde! Par conséquent, la pseudo-objectivité de l'opus cartographique se révèle singulièrement retorse. La carte paraît à la fois actuelle au sens français du terme et actual au sens anglais. Elle est le simulacre d'un état de fait convoquant intemporel et qui s'ancrerait dans un présent aussi extensionnel que celui que décrivait saint Augustin jadis, dans un contexte différent. Elle omet le passé tout en s'efforçant d'imposer le présent. Il lui arrive aussi d'omettre le présent quand elle accorde la priorité au passé (les cartes de la Palestine d'hier et d'aujourd'hui sont pleines de ces glissements, de même qu'au XVIIIe siècle les cartes de la Sicile et d'autres lieux de la Magna Grecia italienne où l'on oblitérait le contemporain afin de renouer avec la toponymie grecque). Il arrive aussi aux cartes de faire abstraction d'un vide momentané pour mieux projeter l'observateur dans un futur virtuel destiné à se réaliser selon les plans préétablis : c'est à cette expérience qu'a été confronté Michael Chabon. En somme, la carte naturalise un flottement, un point de vue erratique qui, toute incrédulité suspendue, ferait passer pour véridique un croquis du monde couvert de texte, de rhombes ou de lignes, d'illustrations, de légendes. Le chemin d'Augustin à Ponce Pilate étant bref à l'aune de l'histoire et des cultures universelles, on répétera la question que posait et se posait le procurateur de Judée: "Quid est veritas?» Difficile de répondre. Et disons le mot, la carte aurait beau s'employer à le faire qu'elle n'y parviendrait pas. Alors, plutôt que de prétendre exposer une véridicité illusoire et, partant, malhonnête, abstenons-nous de formuler des certitudes bien établies, trop vite et trop facilement établies, pour nous contenter d'invoquer une vraisemblance qui ne rend compte que de la semblance du vrai et renonce à la vaine quête du réel monologique.

Cette excursion à travers le monde des cartes comporte quelques enseignements dont la portée est primordiale. Tout d'abord, elle contribue à souligner tout l'intérêt que l'épistémologie de la carte revêt aux yeux des littéraires que nous sommes. Les géographes culturels fréquentent depuis longtemps le domaine des études littéraires; rien ne nous empêche, à nous littéraires, d'effectuer le voyage en sens inverse. Le souhait de confronter l'étude littéraire à d'autres approches du discours portant sur les espaces constitue l'un des moteurs de la géocritique. Car je ne me lasserai jamais d'entonner l'antienne: l'impact de la littérature sur le monde environnant n'est pas négligeable. La prétention de 
la cartographie et, partant, de la géographie, de rendre compte de l'état du monde ne s'accomplit pas dans un pré carré exclusif, soustrait à toute relation de voisinage avec des disciplines ou des discours connexes. La littérature débouche à coup sûr sur une forme d'écriture du réel, son expression esthétique fût-elle ensuite assujettie à des conditions d'interprétation spécifiques. Le géographe et le littéraire participe à l'émergence d'une sorte d'hyper-discours sur le monde. Dans un essai plaçant en regard littérature et cartographie, Peter Turchi écrivait:

L'univers d'une histoire est quelque chose que nous créons ou que nous convoquons mais que le lecteur contribue à créer et à comprendre. Une histoire ou un roman est une sorte de carte car, comme celle-ci, il ne constitue pas un monde mais en évoque un (ou du moins un pour chaque lecteur) ${ }^{10}$.

Le cartographe est un écrivain qui est un cartographe. Le lecteur des cartes est le lecteur d'un roman ou, pour paraphraser le titre d'un livre encore célèbre de Marthe Robert, le lecteur du roman des origines d'une culture. Le monde dans lequel nous vivons est un monde possible, ni plus ni moins que le monde possible que formate le récit du romancier. Et s'il est une différence entre les deux modalités de représentation (et de création) du monde, je dirais que dans l'un heurter un mur entraîne une douleur physique alors que dans l'autre heurter un obstacle éveille d'abord la curiosité de savoir ce qu'il dissimule.

Voilà pour le premier constat. Il en est un autre. Il m'est arrivé d'avoir à présenter les postulats de la méthodologie géocritique. Or, à force de répéter l'exercice, ce qu'il m'incomberait de dire risquerait de se scléroser. Après avoir publié La Géocritique en 2007, j'éprouvais la sensation d'avoir livré le fond de ma pensée. Il m’a été difficile de fournir des commentaires neufs par rapport au livre. Je viens d'achever la rédaction d'un nouvel essai, Le Monde plausible, qui paraîtra chez Minuit à l'automne 2011 et où sera abordée, en diachronie, la dialectique de l'espace ouvert et du lieu clos, qu'arbitre parfois la ligne de l'horizon fabuleux si

Io. P. Turchi, Maps of the Imagination, p. 166: «So the world of a story is a thing we create or summon up into being, but which the reader participates in creating and understanding. A story or novel is a kind of map because, like a map, it is not a world, but it evokes one (or at least one, for each reader).» 
chère à Michel Collot ${ }^{11}$. Ce n'est que maintenant, au sortir de ce travail, qu'il me semble avoir retrouvé le recul indispensable pour pousser un peu plus loin le raisonnement géocritique, car, à mon sens, la géocritique est autant un raisonnement susceptible de déboucher sur une herméneutique qu'une méthodologie. En synthèse, la géocritique se fonde sur trois principes épistémologiques: 1) la spatialité, comme la temporalité, sont tributaires l'une de l'autre et investissent une dimension spatio-temporelle conjointe (l'espace ne saurait par conséquent être examiné hors du temps, et vice-versa) ; 2) loin d'être monologique, lorsqu'elle se veut crédible, la représentation de l'espace est elle-même inspirée de la nature foncièrement mobile de l'objet dont elle se propose de rendre compte; de même que le territoire est toujours saisi dans un processus de déterritorialisation et de reterritorialisation (Gilles Deleuze), de même sa représentation sera transgressive: elle ira outre en permanence; 3) la représentation des espaces que véhiculent les arts et la littérature en particulier donne une leçon sur l'environnement humain car le texte (comme l'image) n'est jamais hors monde: volens nolens, il s'y réfère dans un mouvement entre réel et fiction qui, dans un cadre de "pensée faible" (Gianni Vattimo), ne sont pas les deux pôles antagonistes qu'une certaine critique s'est schématiquement ingéniée à imposer (ah, la textolâtrie!).

L'examen des cartes ou même des simples métaphores cartographiques auxquelles la littérature et les arts visuels recourent avec une insistance croissante (notamment dans les productions issues des aires postcoloniales) peut, me semble-t-il, emprunter un itinéraire géocritique. Car rien n'est plus stratifié qu'une carte, malgré l'emphase qu'elle met sur le présent des lieux - des lieux dont la mise en évidence, la naturalisation (qui attribue une nationalité), constitue l'enjeu innocemment et quelquefois délibérément trompeur. La carte est un palimpseste, comme tout texte, comme toute image. Sa particularité réside dans le fait qu'elle constitue un palimpseste qui élabore dans le temps une vision de l'espace. De ce point de vue, la carte, dans tout ce qu'elle a d'apparemment figé - ne territorialise-t-elle pas à outrance? - est l'expression induite de la transgressivité inhérente aux lieux (et à tous les topoi minoritaires). En un sens, en plus d'un sens, ces lieux ne perdent jamais leur nature d'espace ouvert, car aucune norme - cartographique ou autre - ne saurait immobiliser

II. Voir M. Collot, L'Horizon fabuleux et Paysage et poésie, du romantisme à nos jours (entre autres travaux). 
à tout jamais l'espace en un lieu clos. Enfin, et pour conclure, la carte explicite comme nul autre type de représentation l'ambiguïté du rapport entre réel et fiction: c'est le cours de géographie que les différents écrivains et peintres que j'ai convoqués ici nous auront impartie.

Bertrand WestPHAL

Université de Limoges 


\section{BIBLIOGRAPHIE}

Alpers, Svetlana, "The Mapping Impulse in Dutch Art», in Arts and Cartography. Six Historical Essays, éd. par David Woodward, Chicago/London, The University of Chicago Press, 1987, p. 51-96.

Barthes, Roland, Mythologies, Paris, Seuil, 2010 (1957).

Borges, Jorge Luis, Discusión, Madrid, Alianza Editorial, 1997 (1932).

Bruno, Giuliana, Atlas of Emotion: Journeys in Art, Architecture and Film, London/New York, Verso, 2002.

Снавоn, Michel, Maps and Legends, New York/London, Harper Perennial, 2009.

Collot, Michel, L'Horizon fabuleux, Paris, José Corti, 1988.

—, Paysage et poésie, du romantisme à nos jours, Paris, José Corti, 2005.

Deleuze, Gilles, Parnet, Claire, Dialogues, Paris, Flammarion, 1996.

Friel, Brian, Translations (1981), in Brian Friel: Plays 1, London, Faber and Faber, 1996.

Gruzinski, Serge, La Guerre des images de Christophe Colomb à Blade Runner (1492-2019), Paris, Fayard, 1990.

Marlowe, Christopher, Tamerlan, trad. en français par Philippe de Rothschild, Paris, Albin Michel, 1976.

Peters, Jeffrey N., Mapping Discord. Allegorical Cartography in Early Modern French Writing, Newark, University of Delaware Press, 2004.

Turchi, Peter, Maps of the Imagination. The Writer as Cartographer, San Antonio (TX), Trinity University Press, 2004.

Westphal, Bertrand, La géocritique: réel, fiction, espace, Paris, Les Editions de Minuit, 2007.

—, Le monde plausible: espace, lieu, carte, Paris, Les Editions de Minuit, 2011. 